\title{
Three-dimensional elastic deformation of functionally graded isotropic plates under point loading
}

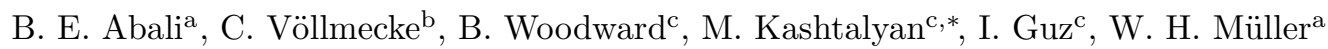 \\ ${ }^{a}$ School V, Institute of Mechanics, Chair of Continuum Mechanics and Materials Theory, Technical University of Berlin, Sekr. MS2, \\ Einsteinufer 5, 10587 Berlin, Germany \\ ${ }^{b}$ School V, Institute of Mechanics, Stability and Failure of Functionally Optimized Structures Group, Sekr. MS2, Einsteinufer 5, 10587 \\ Berlin, Germany \\ ${ }^{c}$ School of Engineering, University of Aberdeen, Fraser Noble Building, Aberdeen AB24 3UE, Scotland, UK
}

\begin{abstract}
In this paper, three-dimensional elastic deformation of isotropic functionally graded plates subjected to point loading is investigated using a combination of analytical and computational means. The analytical approach is based on the displacement functions method, while numerical modeling, which requires high accuracy in the representation of the point loading, uses GALERKIN type finite element method. Three different plate geometries are examined for validation purposes, and the difficulties associated with an optimum choice of the element size are discussed. It is shown that by using a posteriori error estimation based on the equivalent stress measure accurate results can be obtained even in the neighborhood of the point loading.
\end{abstract}

Keywords: Functionally Graded Materials (FGM), variational formulation, finite element method, heterogeneous materials, a posteriori error estimation, adaptive refinement

\section{Introduction}

The concept of Functionally Graded Material (FGM) is currently actively explored in a variety of engineering and biomedical applications where conventional materials can no longer meet increased expectations in terms of performance and structural integrity. FGM refers to advanced composite materials with gradual compositional variation of the constituents from one surface of the material to the other, which results in a continuous variation of material properties. A comprehensive review of the principal development in the modeling of functionally graded materials and structures covering homogenization of particulate FGMs, heat transfer, statics, dynamics, stability fracture, testing and design is given by Birman and Byrd (2007).

\footnotetext{
* Corresponding author

Email address: m.kashtalyan@abdn.ac.uk (M. Kashtalyan)
}

The study of a structure's response to point loading is an important step towards investigation of more complex loading problems including indentation and impact, which requires an understanding of both the local and global response of the material and structure. Much research concentrated on the development of analytical/numerical solutions for isotropic and anisotropic functionally graded half-planes and half-spaces with power-law, exponential and linear variation of the elastic constants with respect to depth, see the survey in Wang et al. (2003). By neglecting boundary dimensions and curvatures, these studies have restricted their interest to the near-field behavior in order to provide a valuable insight into the local stress distribution near the surface of FGMs.

Despite the fact that structures such as beams, plates and shells are frequently subjected to concentrated loads under working or experimental conditions, elastic defor- 
mation of functionally graded structures under point loading has received considerably less attention in the literature. Whilst a number of plate theories for functionally graded plates have been proposed (see e.g., Birman and Byrd (2007)), numerical examples that accompany them are usually restricted to one-term sinusoidal loading and uniformly distributed loading.

A solution to the problem of a concentrated line force acting in the interior of an infinite plate was developed by Spencer (2000). The plate was assumed to be of arbitrary thickness, isotropic and inhomogeneous, with the elastic moduli being functions, not necessarily continuous, of the through-thickness coordinate. The mechanical properties of the plate are not necessarily symmetric about the midsurface. The solution, based on the classical solution for a concentrated force in a thin elastic plate, was extended to give exact closed form solutions for the displacement and stress in the thick inhomogeneous plate.

Guo et al. (2004) examined the interface crack problem for an infinite plate of finite thickness with functionally graded coating subjected to a concentrated force. An exponential variation of the shear modulus in the coating was assumed.

More recently, Woodward and Kashtalyan (2011) investigated the elastic deformation of rectangular sandwich panels with a graded core subjected to various types of localized loads including patch, line, and point load. The elastic behavior of functionally graded rectangular plates under patch load was also studied in Woodward and Kashtalyan (2012). Patches of three different sizes were considered: full-size patch (i.e., uniformly distributed loading), large centralized patch, and small centralized patch. Analytical modeling was based on 3-D elasticity solution for stress and displacement fields in functionally graded plates subjected to a one-term sinusoidal loading recently developed by Kashtalyan (2004), while finite element modeling was performed in ABAQUS with user implemented graded elements. Agreement between the 3-D elasticity solution and finite element model was excellent. It is worth noting that while increasing the number of terms used in the FOURIER representation of patch load type can be seen to give greater accuracy at the plate center, an overshoot in the normal stress is observed in locations of patch load application as the solution tries to capture a step change in load with number of sinusoidal terms. This is known as the GiBBs's phenomenon (Gibbs (1898, 1899)).

Singh and Shukla (2012) performed a nonlinear flexural analysis of simply supported and clamped functionally graded plates under line and point loadings. They employed the LEVINSON shear deformation theory and multiquadratic radial basis functions methods to study the effect of stiffness gradient and boundary conditions on central deflection and in-plane stresses in the plates with power-law variation of YOUNG's modulus through the thickness of the plate.

Sun and Luo (2011b,a, 2012) investigated wave propagation and transient response of functionally graded plates under a point impact loading, while Doddamani et al. (2011) studied the behavior of sandwich beams with functionally graded rubber under three point bending by using a combination of experimental and numerical techniques.

In this paper, the three-dimensional elastic deformation of an FGM rectangular plate subjected to point load is investigated by a combination of analytical and computational tools.

\section{Analytical modeling}

\subsection{Problem formulation}

Consider a rectangular plate of length $a$, width $b$ and thickness $h$. The plate is a three-dimensional continuous body, $\mathcal{B}_{0}$, which is referred to the material configuration expressed in a CARTESIan co-ordinate system $\left(X_{1}, X_{2}, X_{3}\right)$, so that $0 \leq X_{1} \leq a, 0 \leq X_{2} \leq b, 0 \leq X_{3} \leq h$, cf., Fig. 1 .

The material of the FGM plate is assumed to be isotropic inhomogeneous, with an exponential variation of the shear 


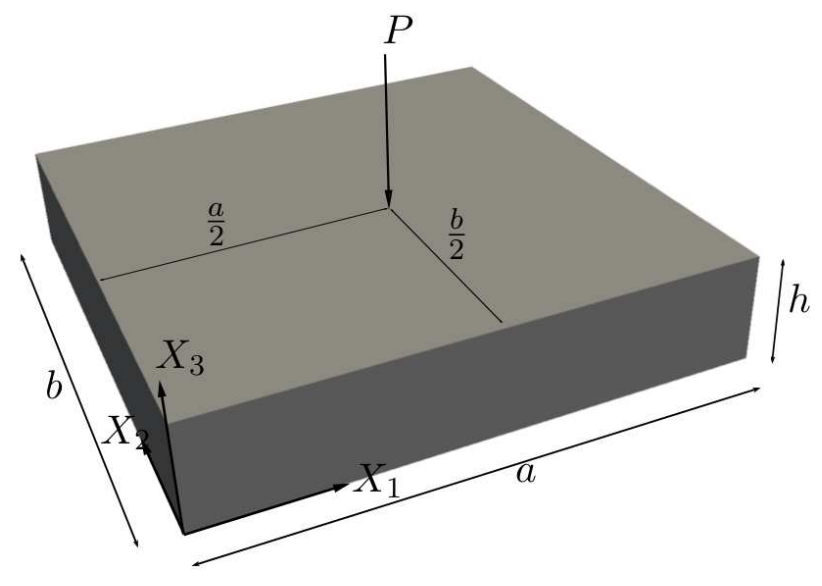

Figure 1: Geometry and loading of the three-dimensional continuum body $\mathcal{B}_{0}$

modulus $G$ with the thickness co-ordinate $X_{3}$ in the form:

$$
\begin{gathered}
G\left(X_{3}\right)=G_{1} \exp \left(\gamma\left(\frac{X_{3}}{h}-1\right)\right), \\
\gamma=\ln \left(\frac{G_{1}}{G_{0}}\right), \nu=\text { const. }
\end{gathered}
$$

Here $G_{0}$ is the value of the shear modulus at the bottom surface of the plate, $X_{3}=0, G_{1}$ is the value of the shear modulus at the top surface of the plate, $X_{3}=h$, and $\gamma$ is the inhomogeneity parameter. The PoIsson's ratio is assumed to be constant.

If the displacement formulation is used, the three-dimensional displacement field in the plate is governed by the following three equilibrium equations in terms of displacements $u_{i}$ :

$$
\begin{gathered}
G \Delta u_{1}+\frac{G}{1-2 \nu} \frac{\partial \varepsilon_{k k}}{\partial X_{1}}+\left(\frac{\partial u_{1}}{\partial X_{3}}+\frac{\partial u_{3}}{\partial X_{1}}\right) \frac{\mathrm{d} G}{\mathrm{~d} X_{3}}=0, \\
G \Delta u_{2}+\frac{G}{1-2 \nu} \frac{\partial \varepsilon_{k k}}{\partial X_{2}}+\left(\frac{\partial u_{2}}{\partial X_{3}}+\frac{\partial u_{3}}{\partial X_{2}}\right) \frac{\mathrm{d} G}{\mathrm{~d} X_{3}}=0, \\
G \Delta u_{3}+\frac{G}{1-2 \nu} \frac{\partial \varepsilon_{k k}}{\partial X_{3}}+\varepsilon_{k k} \frac{\mathrm{d}}{\mathrm{d} X_{3}}\left(\frac{2 G \nu}{1-2 \nu}\right)+ \\
+2 \frac{\partial u_{3}}{\partial X_{3}} \frac{\mathrm{d} G}{\mathrm{~d} X_{3}}=0
\end{gathered}
$$

where and henceforth the summation convention from one to three in repeated indices is applied and the LAPLACEan operator $\Delta=\frac{\partial^{2}}{\partial X_{i} \partial X_{i}}$ as well as the linearized symmetric strains $\varepsilon_{i j}=\frac{\partial u_{(i}}{\partial X_{j}}=\frac{1}{2}\left(\frac{\partial u_{i}}{\partial X_{j}}+\frac{\partial u_{j}}{\partial X_{i}}\right)$ are employed in the usual way. Hence $\varepsilon_{k k}$ is the volumetric strain or dilatation. The above equations are analogous to the NAVIER-LAMÉ equations for homogeneous isotropic materials. In Eq. (2) for the isotropic heterogeneous material or in the NAVIERLAMÉ equations for the isotropic homogeneous case, the same constitutive relation, i.e., HookE's law defines the CAuchy stress tensor:

$$
\sigma_{i j}=\frac{2 G \nu}{1-2 \nu} \varepsilon_{k k} \delta_{i j}+2 G \varepsilon_{i j}
$$

The plate is subjected to a concentrated (point) force, $P$, applied at the center of its top surface, $(a / 2, b / 2, h)$, while the bottom surface remains free, cf., Fig. 1. We will treat the point force as a particular case of distributed transverse loading:

$$
Q\left(X_{1}, X_{2}\right)=Q_{0} \delta\left(X_{1}-X_{1}^{0}\right) \delta\left(X_{2}-X_{2}^{0}\right)
$$

where $Q_{0}=P /(a b)$ and $\delta\left(X_{1}-X_{1}^{0}\right), \delta\left(X_{2}-X_{2}^{0}\right)$ are Deltafunctions such that at $X_{1}=X_{1}^{0}=a / 2, X_{2}=X_{2}^{0}=$ $b / 2$ they be of value one and vanish elsewhere. Then the boundary conditions at the top and bottom surfaces of the plate are

$$
\begin{gathered}
X_{3}=h: \sigma_{33}=Q\left(X_{1}, X_{2}\right), \sigma_{13}=\sigma_{23}=0, \\
X_{3}=0: \sigma_{33}=\sigma_{13}=\sigma_{23}=0 .
\end{gathered}
$$

At the edges of the plate, NAVIER-type boundary conditions are prescribed so that:

$$
\begin{aligned}
& X_{1}=0, X_{1}=a: \sigma_{11}=0, u_{2}=u_{3}=0, \\
& X_{2}=0, X_{2}=b: \sigma_{22}=0, u_{1}=u_{3}=0 .
\end{aligned}
$$

These boundary conditions are representative of roller supports and analogous to simply supported edges used in plate theories.

In order to find the analytical solution to Eq. (2) subject to boundary conditions Eqs. (5),(6), we employ PLEVAKO's displacement potential functions $L=\bar{L}\left(X_{i}\right)$ and $N=\bar{N}\left(X_{i}\right)$. The displacement can be represented in 
terms of the potential functions as:

$$
\begin{gathered}
u_{1}=-\frac{1}{2 G}\left(\nu \Delta-\frac{\partial^{2}}{\partial X_{3}^{2}}\right) \frac{\partial L}{\partial X_{1}}+\frac{\partial N}{\partial X_{2}}, \\
u_{2}=-\frac{1}{2 G}\left(\nu \Delta-\frac{\partial^{2}}{\partial X_{3}^{2}}\right) \frac{\partial L}{\partial X_{2}}+\frac{\partial N}{\partial X_{1}}, \\
u_{3}=-\frac{1}{G}\left(\nu \Delta-\frac{\partial^{2}}{\partial X_{3}^{2}}\right) \frac{\partial L}{\partial X_{3}}+ \\
+\frac{\partial}{\partial X_{3}}\left(\frac{1}{2 G}\left(\nu \Delta-\frac{\partial^{2}}{\partial X_{3}^{2}}\right) L\right) .
\end{gathered}
$$

By referring to HookE's law and to the definition of the strains, the stresses in the plate can be expressed in terms of the displacement potential functions as:

$$
\begin{gathered}
\sigma_{11}=\left(\nu \frac{\partial^{2}}{\partial X_{2}^{2}} \Delta+\frac{\partial^{4}}{\partial X_{1}^{2} \partial X_{3}^{2}}\right) L+2 G \frac{\partial^{2} N}{\partial X_{1} \partial X_{2}}, \\
\sigma_{22}=\left(\nu \frac{\partial^{2}}{\partial X_{1}^{2}} \Delta+\frac{\partial^{4}}{\partial X_{2}^{2} \partial X_{3}^{2}}\right) L-2 G \frac{\partial^{2} N}{\partial X_{1} \partial X_{2}}, \\
\sigma_{33}=\left(\Delta-\frac{\partial^{2}}{\partial X_{3}^{2}}\right)^{2} L \\
\sigma_{13}=-\left(\Delta-\frac{\partial^{2}}{\partial X_{3}^{2}}\right) \frac{\partial^{2} L}{\partial X_{1} \partial X_{3}}+G \frac{\partial^{2} N}{\partial X_{2} \partial X_{3}}, \\
\sigma_{23}=-\left(\Delta-\frac{\partial^{2}}{\partial X_{3}^{2}}\right) \frac{\partial^{2} L}{\partial X_{2} \partial X_{3}}-G \frac{\partial^{2} N}{\partial X_{1} \partial X_{3}}, \\
\sigma_{12}=-\left(\nu \Delta-\frac{\partial^{2}}{\partial X_{3}^{2}}\right) \frac{\partial^{2} L}{\partial X_{1} \partial X_{2}}-G\left(\frac{\partial^{2}}{\partial X_{1}^{2}}-\frac{\partial^{2}}{\partial X_{2}^{2}}\right) N .
\end{gathered}
$$

The displacement functions $L$ and $N$ must satisfy the following partial differential equations:

$$
\begin{gathered}
\Delta\left(\frac{1}{G} \Delta L\right)-\frac{1}{1-\nu}\left(\Delta-\frac{\partial^{2}}{\partial X_{3}^{2}}\right) L \frac{\mathrm{d}^{2}}{\mathrm{~d} X_{3}^{2}}\left(\frac{1}{G}\right)=0, \\
\Delta N+g\left(X_{3}\right) \frac{\partial N}{\partial X_{3}}=0, g\left(X_{3}\right)=\frac{\mathrm{d}}{\mathrm{d} X_{3}}\left(\ln \left(G\left(X_{3}\right)\right)\right) .
\end{gathered}
$$

\subsection{Method of solution}

Given the boundary conditions at the edges of the plate, we will expand the transverse loading $Q\left(X_{1}, X_{2}\right)$ into a double FouRIER series as:

$$
Q\left(X_{1}, X_{2}\right)=-\sum_{m=1}^{\infty} \sum_{n=1}^{\infty} q_{m n} \sin \left(\frac{\pi m X_{1}}{a}\right) \sin \left(\frac{\pi n X_{2}}{b}\right)
$$

where $m$ and $n$ are wave numbers, and the loading coefficient $q_{m n}$ is equal to

$$
\begin{gathered}
q_{m n}=\frac{2}{a} \int_{0}^{a} \frac{2}{b} \int_{0}^{b} Q\left(X_{1}, X_{2}\right) \sin \left(\frac{\pi m X_{1}}{a}\right) \times \\
\times \sin \left(\frac{\pi n X_{2}}{b}\right) \mathrm{d} X_{1} \mathrm{~d} X_{2}= \\
=\frac{4 P}{a b} \sin \left(\frac{\pi m X_{1}^{0}}{a}\right) \sin \left(\frac{\pi n X_{2}^{0}}{b}\right)= \\
=\frac{4 P}{a b} \sin \left(\frac{\pi m}{2}\right) \sin \left(\frac{\pi n}{2}\right), m, n=1,3,5, \ldots
\end{gathered}
$$

Consequently, the displacement potential function will be sought in the form:

$$
\begin{aligned}
& \bar{L}\left(X_{i}\right)=\sum_{m=1}^{\infty} \sum_{n=1}^{\infty} \psi_{1 m n}\left(X_{1}, X_{2}\right) \phi_{1 m n}\left(X_{3}\right) \\
& \bar{N}\left(X_{i}\right)=\sum_{m=1}^{\infty} \sum_{n=1}^{\infty} \psi_{2 m n}\left(X_{1}, X_{2}\right) \phi_{2 m n}\left(X_{3}\right) .
\end{aligned}
$$

Then, for each pair of $m$ and $n$ the Eqs. (10) are transformed into the following four differential equations:

$$
\begin{gathered}
\frac{\partial^{2} \psi_{i m n}}{\partial X_{1}^{2}}+\frac{\partial^{2} \psi_{i m n}}{\partial X_{2}^{2}}+\alpha_{m n}^{2} \psi_{i m n}=0, \quad i=1,2, \\
\frac{\mathrm{d}^{4} \phi_{1 m n}}{\mathrm{~d} X_{3}^{2}}-2 g\left(X_{3}\right) \frac{\mathrm{d}^{3} \phi_{1 m n}}{\mathrm{~d} X_{3}^{3}}+\left(g^{2}\left(X_{3}\right)-\frac{\mathrm{d} g\left(X_{3}\right)}{\mathrm{d} X_{3}}-\right. \\
\left.-2 \alpha_{m n}^{2}\right) \frac{\mathrm{d}^{2} \phi_{1 m n}}{\mathrm{~d} X_{3}^{2}}+2 \alpha_{m n}^{2} g\left(X_{3}\right) \frac{\mathrm{d} \phi_{1 m n}}{\mathrm{~d} X_{3}}+ \\
+\alpha_{m n}^{2}\left(\alpha_{m n}^{2}+\frac{\nu}{1-\nu}\left(g^{2}\left(X_{3}\right)-\frac{\mathrm{d} g\left(X_{3}\right)}{\mathrm{d} X_{3}}\right)\right) \phi_{1 m n}=0, \\
\frac{\mathrm{d}^{2} \phi_{2 m n}}{\mathrm{~d} X_{3}^{2}}+g\left(X_{3}\right) \frac{\mathrm{d} \phi_{2 m n}}{\mathrm{~d} X_{3}}-\alpha_{m n}^{2} \phi_{2 m n}=0,
\end{gathered}
$$

where $\alpha_{m n}=\pi \sqrt{\left(\frac{m}{a}\right)^{2}+\left(\frac{n}{b}\right)^{2}}$. For a simply supported functionally graded rectangular plate with the dependence of the shear modulus on the thickness co-ordinate in the exponential relation as in Eq. (1), the functions $\psi_{1 m n}, \psi_{2 m n}$, $\phi_{1 m n}$, and $\phi_{2 m n}$, in Eqs. (10) are found to be

$$
\begin{aligned}
& \psi_{1 m n}\left(X_{1}, X_{2}\right)=\sin \left(\frac{\pi m X_{1}}{a}\right) \sin \left(\frac{\pi n X_{2}}{b}\right), \\
& \psi_{2 m n}\left(X_{1}, X_{2}\right)=\cos \left(\frac{\pi m X_{1}}{a}\right) \cos \left(\frac{\pi n X_{2}}{b}\right),
\end{aligned}
$$




$$
\begin{gathered}
\phi_{1 m n}\left(X_{3}\right)=q_{m n} h^{4} \exp \left(\frac{\gamma X_{3}}{h}\right)\left(A_{1 m n} \times\right. \\
\times \cosh \left(\frac{\lambda_{m n} X_{3}}{h}\right) \cos \left(\frac{\mu_{m n} X_{3}}{h}\right)+ \\
+A_{2 m n} \sinh \left(\frac{\lambda_{m n} X_{3}}{h}\right) \cos \left(\frac{\mu_{m n} X_{3}}{h}\right)+ \\
+A_{3 m n} \cosh \left(\frac{\lambda_{m n} X_{3}}{h}\right) \sin \left(\frac{\mu_{m n} X_{3}}{h}\right)+ \\
\left.+A_{4 m n} \sinh \left(\frac{\lambda_{m n} X_{3}}{h}\right) \sin \left(\frac{\mu_{m n} X_{3}}{h}\right)\right) \\
\phi_{2 m n}\left(X_{3}\right)=\frac{q_{m n} h^{2}}{G_{1}} \exp \left(-\frac{\gamma X_{3}}{h}\right)\left(A_{5 m n} \times\right. \\
\times \cosh \left(\frac{\beta_{m n} X_{3}}{h}\right)+ \\
\left.+A_{6 m n} \sinh \left(\frac{\beta_{m n} X_{3}}{h}\right)\right)
\end{gathered}
$$

where

$$
\begin{gathered}
\left(\begin{array}{c}
\lambda_{m n} \\
\mu_{m n}
\end{array}\right)=\sqrt{\frac{1}{2}\left( \pm \beta_{m n}^{2}+\sqrt{\beta_{m n}^{4}+\gamma^{2} \alpha_{m n}^{2} h^{2} \frac{\nu}{1-\nu}}\right)} \\
\beta_{m n}=\sqrt{\frac{\gamma^{2}}{4}+\alpha_{m n}^{2} h^{2}}
\end{gathered}
$$

By substituting the functions $\psi_{1 m n}, \psi_{2 m n}, \phi_{1 m n}$, and $\phi_{2 m n}$ and Eqs. (17) into Eqs. (13), and by using Eqs. (11),(12) the following representation for the displacements and stresses in the plate is obtained:

$$
\begin{aligned}
u_{1}= & \sum_{m=1}^{\infty} \sum_{n=1}^{\infty} \sum_{k=1}^{6} A_{k m n} U_{1, k m n}\left(X_{3}\right) \times \\
& \times \cos \left(\frac{\pi m X_{1}}{a}\right) \sin \left(\frac{\pi n X_{2}}{b}\right), \\
u_{2}= & \sum_{m=1}^{\infty} \sum_{n=1}^{\infty} \sum_{k=1}^{6} A_{k m n} U_{2, k m n}\left(X_{3}\right) \times \\
& \times \sin \left(\frac{\pi m X_{1}}{a}\right) \cos \left(\frac{\pi n X_{2}}{b}\right), \\
u_{3}= & \sum_{m=1}^{\infty} \sum_{n=1}^{\infty} \sum_{k=1}^{6} A_{k m n} U_{3, k m n}\left(X_{3}\right) \times \\
& \times \sin \left(\frac{\pi m X_{1}}{a}\right) \sin \left(\frac{\pi n X_{2}}{b}\right),
\end{aligned}
$$

$$
\begin{aligned}
& \sigma_{\underline{j} \underline{j}}= \sum_{m=1}^{\infty} \sum_{n=1}^{\infty} \sum_{k=1}^{6} A_{k m n} P_{\underline{j} \underline{j}, k m n}\left(X_{3}\right) \times \\
& \times \sin \left(\frac{\pi m X_{1}}{a}\right) \sin \left(\frac{\pi n X_{2}}{b}\right), j=1,2,3, \\
& \sigma_{13}=\sum_{m=1}^{\infty} \sum_{n=1}^{\infty} \sum_{k=1}^{6} A_{k m n} P_{13, k m n}\left(X_{3}\right) \times \\
& \times \cos \left(\frac{\pi m X_{1}}{a}\right) \sin \left(\frac{\pi n X_{2}}{b}\right), \\
& \sigma_{23}=\sum_{m=1}^{\infty} \sum_{n=1}^{\infty} \sum_{k=1}^{6} A_{k m n} P_{23, k m n}\left(X_{3}\right) \times \\
& \times \sin \left(\frac{\pi m X_{1}}{a}\right) \cos \left(\frac{\pi n X_{2}}{b}\right), \\
& \sigma_{12}= \sum_{m=1}^{\infty} \sum_{n=1}^{\infty} \sum_{k=1}^{6} A_{k m n} P_{12, k m n}\left(X_{3}\right) \times \\
& \times \cos \left(\frac{\pi m X_{1}}{a}\right) \cos \left(\frac{\pi n X_{2}}{b}\right) .
\end{aligned}
$$

The constant coefficients $A_{k m n}$ are determined from boundary conditions at the top and bottom surfaces of the plate, Eqs. (5),(6). The functions $U_{i, k m n}$ for the displacements and for stresses $P_{i, k m n}$ are given in the appendix.

\section{Numerical modeling}

In contrast to the analytic formulations presented in the preceding section numerical modeling of functionally graded materials allows us to investigate more complex geometries and phenomena. Since the representation of a point load is difficult computationally, the consistency and the quality of the numerical modeling can be verified by using the analytic solution for the point loading. The analytic solution outlined in the preceding section assumes $C^{\infty}$ continuity for the displacement functions $\boldsymbol{u}$ in space $\boldsymbol{X}$. This is usually the case in continuum mechanics and thus not mentioned. In a discrete numerical approach such as the finite element method, however, the continuity of the solution depends on the function space. Any function $\psi(\boldsymbol{X}, t)$, defined in space and time, is decomposed into a time dependent and a space dependent function:

$$
\psi(\boldsymbol{X}, t)=\sum_{\mathrm{ID}} \Psi^{\mathrm{ID}}(t) \phi^{\mathrm{ID}}(\boldsymbol{X})
$$


Summation is applied over the nodal points with an identification number, ID. The space function $\phi^{\mathrm{ID}}(\boldsymbol{X})$ determines the geometric configuration, i.e., the connectivity between the neighboring nodes by using form functions of any polynomial degree stated by the function space, as described in Hilbert (1902). Formally Eq. (24) is a discrete representation of $\psi(\boldsymbol{X}, t)$ in a finite dimensional subspace of the Sobolev space, $\mathcal{H}^{p}$. Such a space $\mathcal{H}^{p}$ can be of any polynomial degree $p$. The time function $\Psi^{\mathrm{ID}}(t)$ represents the values of the function $\psi(\boldsymbol{X}, t)$ in each node. For a static analysis, which we will implement herein, the time function possesses only one set of values for the deformation. For a dynamic analysis it would have one set of values for each time step. Every set includes discrete values in the nodes, which form a continuous field interpolated with form functions of polynomial degree $p$. Therefore, the accuracy of the computation with the same amount of nodes depends on the form functions used in the formulation. The field, however, is discrete with a support of one element, i.e., the distribution within the element does not affect any other element and, moreover, the continuity across the elements is of order 1 without depending on the degree of chosen functional space. The objective is to find the displacements in the three directions in space. Thus we have three different functions to discretize as given in Eq. (24). Herein we will use quadratic elements for the displacements, i.e., $u^{i} \in \mathcal{H}^{2}$, that induce linear stress distribution, which is discontinuous across elements. The implementation with linear elements is realized in Abali et al. (2012). We shall give a brief outline of the variational formulation and implement the point load next.

Starting in a known reference state, $\mathcal{B}_{0}$, a continuum body is deformed into $\mathcal{B}$ subject to given loading conditions. We want to calculate the displacement field $u^{i}$ of every particle referring to the state $\mathcal{B}_{0}$. If a particle originally at $X^{i}$ moves to $x^{i}$, the displacement of that particle is $u^{i}=x^{i}-X^{i}$. The used numerical integral calculation, i.e., GAUssian quadrature, is the easiest in an orthonormal coordinate system, thus, $X^{i}$ and $u^{i}$ are expressed in CARTEsIan coordinates. The continuum is not deformed initially, thus the geometry at the beginning provides a known state, which we employ as the reference state. Since the reference, thus the initial state, is expressed in CARTESIan coordinates, the deformed state is in curvilinear coordinates where the mapping between these two states is given by the deformation gradient $F^{i}{ }_{j}=$ $\partial x^{i} / \partial X^{j}$. Under the assumption of small displacements, i.e., $\partial x^{i} / \partial X^{j} \approx \delta^{i}{ }_{j}$, the balance of mass and linear momentum in the deformed state reads

$$
\begin{gathered}
\frac{\mathrm{d}}{\mathrm{d} t} \int_{\mathcal{B}} \rho \mathrm{d} v=0 \\
\frac{\mathrm{d}}{\mathrm{d} t} \int_{\mathcal{B}} \rho v^{i} \mathrm{~d} v=\int_{\partial \mathcal{B}} \sigma^{j i} \mathrm{~d} a_{j}+\int_{\mathcal{B}} \rho f^{i} \mathrm{~d} v .
\end{gathered}
$$

They can be mapped on to the initial state

$$
\begin{gathered}
\rho=\rho_{0} J \\
\int_{\mathcal{B}_{0}} \rho_{0} \frac{\partial v^{i}}{\partial t} \mathrm{~d} V=\int_{\partial \mathcal{B}_{0}} \sigma^{j i} \delta^{r}{ }_{j} \mathrm{~d} A_{r}+\int_{\mathcal{B}_{0}} \rho_{0} f^{i} \mathrm{~d} V
\end{gathered}
$$

where the mass density in actual and initial frame $\rho$ and $\rho_{0}$, respectively, velocity field $v^{i}$, specific body forces $f^{i}$ and the CAUCHY stress tensor $\sigma^{j i}$, are transformed onto the initial state by using the JACoBIan $J=\operatorname{det}\left(F^{i}{ }_{j}\right) \approx 1$. We employed the volume and surface element transformations, $\mathrm{d} v=J \mathrm{~d} V \approx \mathrm{d} V, \mathrm{~d} a_{i}=\left(\frac{\partial x^{i}}{\partial X^{r}}\right)^{-1} J \mathrm{~d} A_{r} \approx \delta^{r}{ }_{i} \mathrm{~d} A_{r}$, cf., (Müller, 2011, p. 62) so that the Eq. (26) holds in general for all closed (subject to mass transfer) systems. For our problem, however, first, we neglect the inertia term $\rho_{0} \partial v^{i} / \partial t$ since an elastic deformation is supposed to be instantaneous. Second, body forces, such as gravity $\rho_{0} f^{i}$, are ignored because the deformation due to its own weight is supposed to be small with respect to the deformation subject to traction on the surface. If continuity of stress within the body is fulfilled then GAUss's theorem can be applied so that the latter formulation yields to the so- 
called equilibrium condition:

$$
0=\int_{\partial \mathcal{B}_{0}} \sigma^{r i} \mathrm{~d} A_{r}=\int_{\mathcal{B}_{0}} \frac{\partial \sigma^{r i}}{\partial X^{r}} \mathrm{~d} V \Rightarrow \sigma_{, j}^{j i}=0
$$

Of course the continuity of stress is not satisfied in the discretized form. Therefore, the latter equation will be written for each element, where the stress is linear and then summed up. Since the formulation is in the initial state, where we evaluate using CARTESIan coordinates, the co-and contravariant distinction is superfluous and will be suppressed henceforth. The set of trial (ansatz) functions $u_{i}$ are searched in the discretized space and we do not distinguish between discretized and continuous functions in the notation. In order to apply a variational formulation we shall multiply the Eq. (27) with tensor of appropriate rank. This can be easily associated with an arbitrary test function $\delta u_{i}$. We choose it from the same $\mathcal{H}^{2}$-space as $u_{i}$, also known as the GALERKIN method in finite element analysis, such that the test functions of length one are defined by using the same connectivity as in Eq. (24):

$$
\delta u_{i}=\sum_{\mathrm{ID}} 1_{i}^{\mathrm{ID}} \phi^{\mathrm{ID}}\left(X^{i}\right)
$$

After integration by parts and the implementation of $\sigma_{j i} N_{j}=$ $\hat{t}_{i}$ on the boundaries the variational form to be computed reads

$$
\begin{gathered}
0=\int_{\mathcal{B}_{0}} \sigma_{j i, j} \delta u_{i} \mathrm{~d} V=-\int_{\mathcal{B}_{0}} \sigma_{j i} \delta u_{i, j} \mathrm{~d} V+ \\
+\int_{\partial \mathcal{B}_{\mathrm{N}}} \hat{t}_{i} \delta u_{i} \mathrm{~d} A
\end{gathered}
$$

As in the analytical formulation, cf., Eqs. (5), (6), the boundary conditions on $\partial \mathcal{B}_{\mathrm{D}}$ boundaries will be applied strongly. By using the Eq. (3) the variational form (29) is computed such that the integral equation is minimized. All preprocessing and programming, as well as computation with iterative methods ${ }^{1}$ has been obtained by using the

\footnotetext{
${ }^{1}$ We have used GMRES from Trilinos solvers project of Sandia National Lab., cf., http://trilinos.sandia.gov/, via single $3003.0 \mathrm{MHz}$ Intel $^{\mathrm{TM}}$ processor in Ubuntu ${ }^{\mathrm{TM}}$ operating system
}

open-source package collection in the FEniCS project, see Logg et al. (2011); Hoffman et al. (2005); Logg and Wells (2010). The visualization is undertaken with MatPlotLIB, see Hunter (2007) and Paraview, see Henderson (2007). The code is supplied in our website, see Abali (2011-) under the GNU public license in Gnu Public (2007).

The variational formulation above is well-known. For a convergence study with heterogeneous materials we refer to Abali et al. (2012). In the following we discretize the domain automatically with an approximate global size for each element. The series representation of the traction is discussed and improved and results are amended by an adaptive meshing algorithm as will be discussed in $\S 5$.

\section{Validation and results}

The numerical formulation presented in the previous section is validated by using the analytical solution presented in $\S 2$. To find the analytical solution, a concentrated (point) load applied at the top surface of the plate was treated as a particular case of distributed transverse loading, Eq. (4), and then represented as an infinite series, Eq. (11). In order for the analytical solution to be computed, the series has to be truncated after a finite number of terms. In this study, the number of terms in the FOURIER representation of the point load is taken as 50 . Therefore, the point load, i.e., the traction reads

$$
\begin{gathered}
\hat{t}_{i}=\left(\begin{array}{c}
0 \\
0 \\
\hat{Q}
\end{array}\right), \\
\hat{Q}=-\sum_{m=1}^{50} \sum_{n=1}^{50} \frac{4 P}{a b} \sin \left(\frac{\pi m}{2}\right) \sin \left(\frac{\pi n}{2}\right) \times \\
\times \sin \left(\frac{\pi m X_{1}}{a}\right) \sin \left(\frac{\pi n X_{2}}{b}\right) .
\end{gathered}
$$

This finite series models a point load as a highly localized distributed loading of a limited magnitude. The magnitude of the loading as well as the size of the area upon which it is concentrated is set by the number of terms 

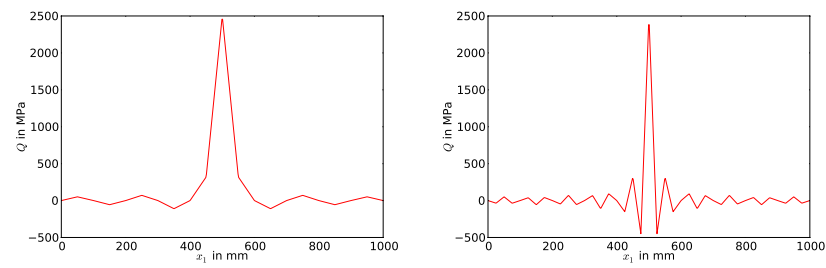

Figure 2: Magnitude of the point loading projected onto mesh of 20 and 40 elements in one direction.

in the series. Especially around the origin, where the magnitude of loading is the highest, the representation of the series depends heavily on the underlying triangulation (mesh). In order to see this effect the traction is projected onto different meshes of constant element size (Fig. 2). Increasing the number of terms in the FourieR representation will lead to the load concentrated over an area of decreasing size. However, the solution will not converge globally due to GiBBS's phenomenon, see Gibbs $(1898,1899)$. In order to circumvent any numerical problems due to GiBBs's phenomenon, we propose to use a GAUSSian distribution function such that

$$
\hat{Q}=\frac{-q_{0}}{\sqrt{2 \pi \sigma^{2}}} \exp \left(\frac{1}{2 \sigma^{2}}\left(-\left(X_{1}-\frac{a}{2}\right)^{2}-\left(X_{2}-\frac{b}{2}\right)^{2}\right),\right.
$$

where the amplitude $q_{0}=50000 \mathrm{MPa}$ and the variance $\sigma=8$ have been determined from inspection to match the truncated series representation with $P /(a b)=Q_{0}=1$ $\mathrm{MPa}$ as shown in Fig. 3.

The results are presented for three plates whose geometrical and material parameters are given in Table 1 . Plates 1 and 2 have the same dimensions and thickness but opposite stiffness gradients, with the inhomogeneity parameters $\gamma$ being of the same absolute magnitude but opposite signs. In plate 1, Young's modulus decreases exponentially from the value of $200 \mathrm{GPa}$ at the top surface of plate to the value of $70 \mathrm{GPa}$ at the bottom surface. Plate 2 can be viewed as a "flipped over" plate, with Young's modulus increasing exponentially from the value of $70 \mathrm{GPa}$ at the top surface to the value of $200 \mathrm{GPa}$ at the bottom surface of the plate. Plates 1 and 3 have the same length and

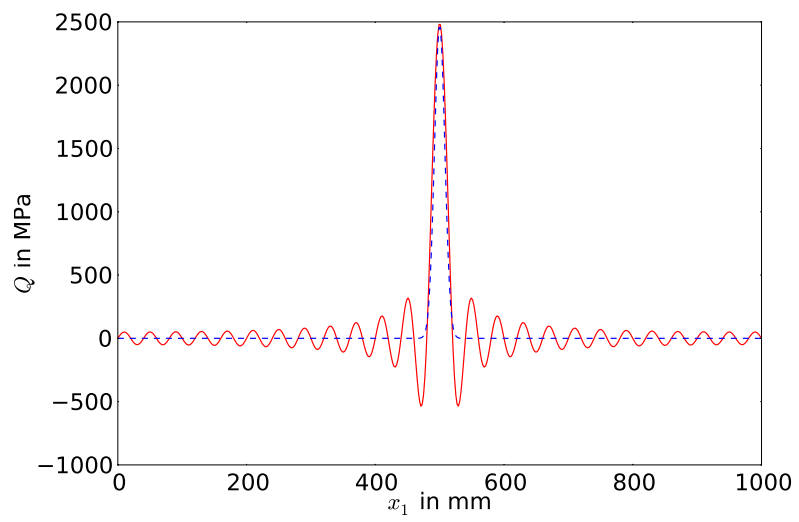

Figure 3: Magnitude of the point loading is depicted in one direction: the series representation as in Eq. (30) in redcontinuous line and the Gaussian function as in Eq. (31) in blue-dashed line.

width as well as the stiffness gradient but different thickness. Plate 1 is a thick plate with $a / h=b / h=3$, while plate 3 is a thin plate with $a / h=b / h=10$.

Table 2 shows computational times required for numerical modelling of plate 2 using automatic meshing with constant element size. The system is solved for displacements. After that the stresses are computed by using HookE's law. Therefore, two subsequent computations with different numbers of degrees of freedom are utilised resulting in long computational times. It is evident that although more elements would give more accurate results, automatic meshing is not feasible in such extreme loading cases. In the computations shown below, 40 elements in $X_{1}$ and $X_{2}$ directions (Fig. 1) are used. The number of elements in $X_{3}$ direction is such that the element size is equal in each direction. The displacement field is in $\mathcal{H}^{2}$ so that the stress field is in $\mathcal{H}^{1}$ and discontinuous across the elements. Figure 4 shows through thickness variation of normalized stresses $\bar{\sigma}_{i j}=\sigma_{i j} / Q_{0}$ in plate 1 (reference plate) and plate 2 ("flipped over" plate). The in-plane normal stress $\bar{\sigma}_{11}$ (Fig. 4(a)) and the out-of-plane normal stress $\bar{\sigma}_{33}$ (Fig. 4(b)) are shown at the center of the plate $\left(a / 2, b / 2, X_{3}\right)$, while the in-plane shear stress $\bar{\sigma}_{12}$ (Fig. 4(c)) and the transverse (out-of-plane) shear stress $\bar{\sigma}_{13}$ (Fig. $\left.4(\mathrm{~d})\right)$ are shown at the $\left(0,0, X_{3}\right)$ and $\left(0, b / 2, X_{3}\right)$ 


\begin{tabular}{c|c|c|c|c|c|c|c} 
Plate & $E_{\text {top }}[\mathrm{GPa}]$ & $E_{\text {bottom }}[\mathrm{GPa}]$ & $\nu[-]$ & $\gamma[-]$ & $a[\mathrm{~mm}]$ & $b[\mathrm{~mm}]$ & $h[\mathrm{~mm}]$ \\
\hline 1 & 200 & 70 & 0.3 & 1.04982 & 1000 & 1000 & 300 \\
2 & 70 & 200 & 0.3 & -1.04982 & 1000 & 1000 & 300 \\
3 & 200 & 70 & 0.3 & 1.04982 & 1000 & 1000 & 100 \\
\hline
\end{tabular}

Table 1: Geometry and material details of the plates.

\begin{tabular}{c|c|c|c|c}
$\begin{array}{c}\text { Nr. of elements } \\
\text { in } X_{1} \text { and } X_{2} \text { directions }\end{array}$ & $\begin{array}{c}\text { DOFs of } \\
\text { displacements }\end{array}$ & $\begin{array}{c}\text { DOFs of } \\
\text { stresses }\end{array}$ & $\begin{array}{c}\text { computation time of } \\
\text { displacements and stresses [min] }\end{array}$ & \\
\hline 10 & 9,261 & 64,800 & 0.205 & \\
20 & 65,559 & 518,400 & 1.333 & \\
40 & 531,441 & $4,492,800$ & 624.247 & \\
\hline
\end{tabular}

Table 2: Computational times for plate 2 using automatic meshing with constant element size.

edges of the plate, respectively. Note that according to Eq. (23), the shear stresses are equal to zero at the center of the plate. From comparison of the numerical and analytical approaches it is evident that the mesh is not quantitatively sufficient for capturing the distribution of normal stresses at the center of plate directly below the site of load application accurately. At the same time, away from the plate center, good agreement between analytical and numerical approaches is observed for the shear stresses $\bar{\sigma}_{12}$. The difference between analytical and numerical predictions for $\bar{\sigma}_{13}$ can be attributed to the difference in representation of the point load within the analytical and numerical solutions, aforementioned in Eqs. (30) and (31), visualized in Fig. 3. Comparison of plates 1 and 2 also shows that reversing the direction of stiffness grading (i.e., changing the sign of the inhomogeneity parameter $\gamma$ ) does not have any significant effect on the distribution of normal stresses directly underneath the point of load application (Fig.4(a),(b)). However it has an effect on the distribution of shear stresses (Fig. 4(c),(d)).

Figure 5 shows the through thickness variation of normalized stresses $\bar{\sigma}_{i j}=\sigma_{i j} / Q_{0}$ in plate 1 (thick plate) and plate 3 (thin plate). It can be seen that in plate 3 (thin plate) as opposed to plate 1 (thick plate) the inplane normal stresses $\bar{\sigma}_{11}$ are still present at the bottom surface of the plate (Fig. $5(\mathrm{a})$ ). This can be attributed to the overall bending of the plate under the point load.
Also, it can be observed that the shear stresses in plate 3 (thin plate) are significantly higher than in plate 1 (thick plate), (Fig. 5(c),(d)). This is consistent with the results obtained by Pagano (1969) who constructed the exact solution for a plate within linear elasticity theory and compared them to the corresponding classical plate theory solutions. According to the KIRCHHOFF-Love hypothesis, on which the classical plate theory is based, the normal to the mid-plane is assumed to remain straight and normal after deformation. This is equivalent to assuming that the shear strains in the planes normal to the mid-plane are equal to zero. This leads to neglecting transverse shear stresses within the classical plate theory in a sense that they cannot be determined from the constitutive equations as a consequence of the KIRCHHOFF-LOVE hypothesis. Despite this deficiency, classical plate theory delivers reasonable results for displacements and in-plane stresses for plates with length-to-thickness ratio greater than 20 . Pagano (1969) showed that transverse shear stresses computed by integration of the equilibrium equations using the in-plane stresses obtained within the classical plate theory are in good agreement with stresses predicted by the three-dimensional elasticity theory for a single-layered plate with length-to-thickness ratio equal to 4 and a threelayered plate with length-to-thickness ratio equal to 10 . The difference between analytical and numerical predictions for $\bar{\sigma}_{13}$ is also more pronounced in plate 3 (thin plate) 

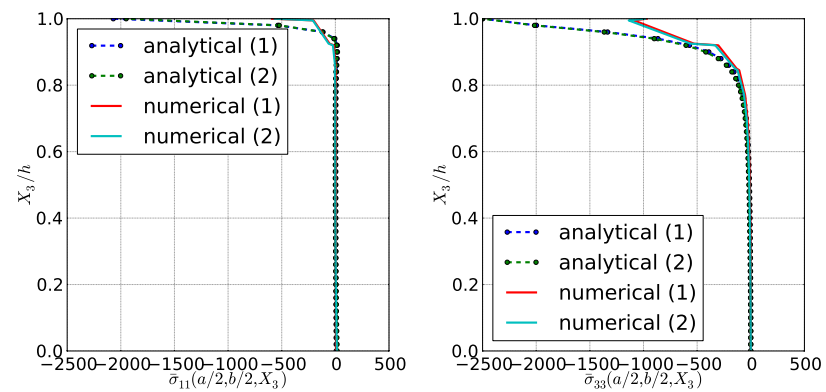

(a) (in-plane) normal stress $\bar{\sigma}_{11}$ (b) (out-of-plane) normal stress $\bar{\sigma}_{33}$
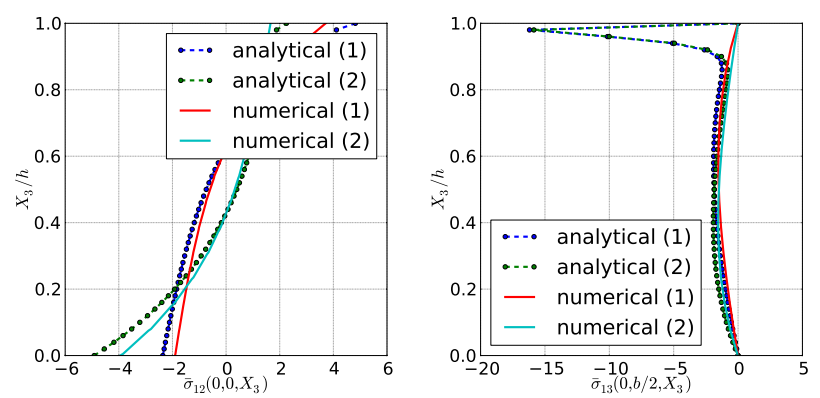

(c) (in-plane) shear stress $\bar{\sigma}_{12}$

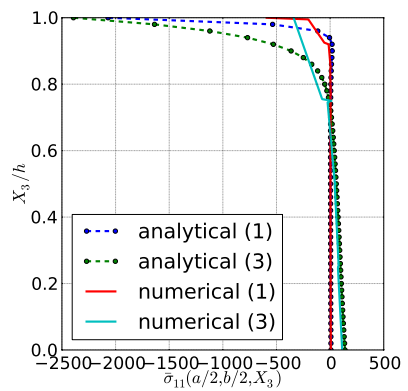

(a) (in-plane) normal stress $\bar{\sigma}_{1}$

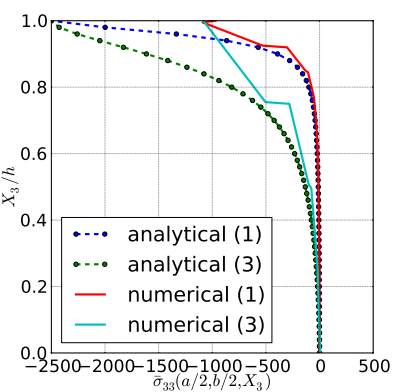

(b) (out-of-plane) normal stress $\bar{\sigma}_{33}$

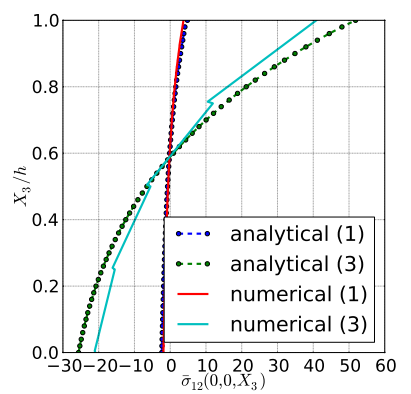

(c) (in-plane) shear stress $\bar{\sigma}_{12}$

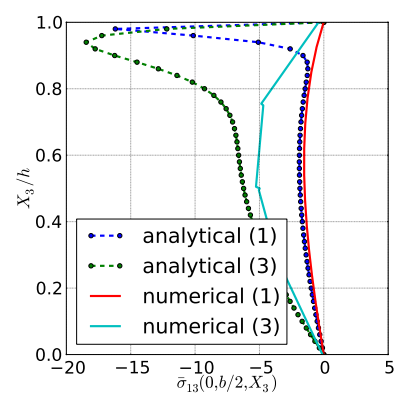

(d) (out-of-plane) shear stress $\bar{\sigma}_{13}$

Figure 4: Through thickness variation of the normalized stresses $\bar{\sigma}_{i j}$ in Plate 1 (reference plate) and Plate 2 "flipped over" plate) according to numerical solution (solid lines) and analytical solution (dashed lines)

than in plate 1 (thick plate) and can be attributed to the difference in representation of the point load within the analytical and numerical solutions, as mentioned above.

\section{Error estimation and adaptive-refinement}

For an approximated numerical solution the error cannot be calculated unless there exists an exact solution. For a generic case, since we do not have an analytic solution, the error can be estimated regarding a measure upon the numerical solution, i.e., the displacement field $u_{i}$. Since for engineering purposes the stress is the parameter governing structural analysis, a posteriori error estimation based on the stress tensor seems to be appropriate. Thus, an equivalent stress, such as the vON MisEs equivalent stress, $\sigma^{\mathrm{eq}}$,

Figure 5: Through thickness variation of normalized stresses $\bar{\sigma}_{i j}$ in Plate 1 (thick plate) and Plate 3 (thin plate) according to numerical solution (solid lines) and analytical solution (dashed lines).

is chosen:

$$
\sigma^{\mathrm{eq}}=\sqrt{\frac{3}{2} \sigma_{\langle i j\rangle} \sigma_{\langle i j\rangle}}, \sigma_{\langle i j\rangle}=\sigma_{i j}-\frac{1}{3} \sigma_{k k} \delta_{i j} .
$$

Theoretically, point loading evokes infinite stresses at the point where the load is applied. Even if the load was imposed on one single node discrete interpolation would spread it to the elements sharing that node. Herein, we use a finite series for the loading and Eq. (31) for implementing it. Thus, infinite stresses will not be produced but the maximum value of the equivalent stress occurs where the load has its highest value - in the center of the top surface of the plate. Therefore, we propose to use the value of the equivalent stress in the whole domain in order to estimate whether the mesh requires refinement or not. Since the maximum stress occurs in the center of the top surface of the plate the number of elements next 
to it will be increased and the transition to lower stress will be smoothed. The maximum stress value is computed automatically, not in a given coordinate so that the proposed approach is generic. In order to determine which element should be refined the elements having equivalent stress in their midpoints higher than a given limit value are marked. The limit value is obtained as a fraction of the maximum equivalent stress value in the whole plate. $10 \%$ of the maximum equivalent stress is set to be the lower limit, and all elements above that limit are marked. The marked elements are refined automatically without any hanging nodes using the FEniCS project (Logg et al., $2011, \S 29)$. By starting off with a coarse mesh this adaptive refinement technique has been used iteratively, such that each time the numerical solution is used for a posteriori error estimation based on the equivalent stress. This is repeated fifteen times and Fig. 6 shows the mesh and its positive effect on the solution as well as the magnitude of the displacements as a color distribution.
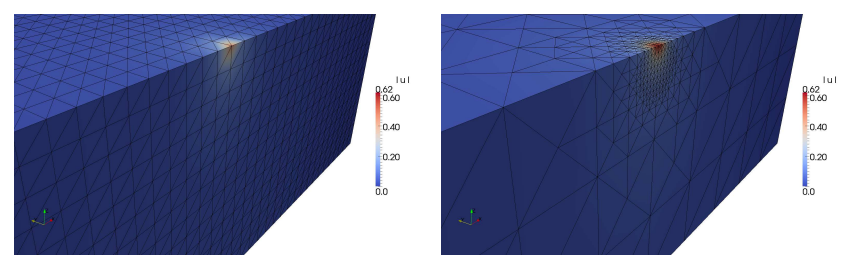

Figure 6: Distribution of the magnitude of the displacement on plate 2 and the underlying mesh left: after a constant size refinement $(531,441$ DOFs), right: after adaptive refinement $(99,675$ DOFs).

The adaptive refinement significantly increases the accuracy of the results in the normal stresses near to the point loading for all three cases considered. This is apparent when comparing the normal in-plane stress distribution $\bar{\sigma}_{11}$ before and after adaptive refinement, cf., Fig. 4 (a) and Fig. 7 (a) for plate 1 and 2 and Fig. 5 (a) and Fig. 7 (b) for comparison of plate 1 and 3, respectively. Furthermore, the improvement of the distribution of the out-of-plane stress $\bar{\sigma}_{33}$ can be noted in Fig. 4 (b) and Fig. 8 (a) for plate 1 and 2 and Fig. 5 (b) and Fig. 8 (b) for plate 1 and 3 , respectively.

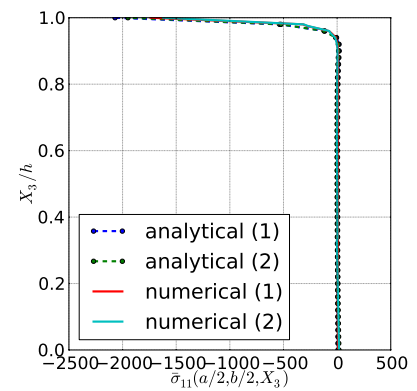

(a)

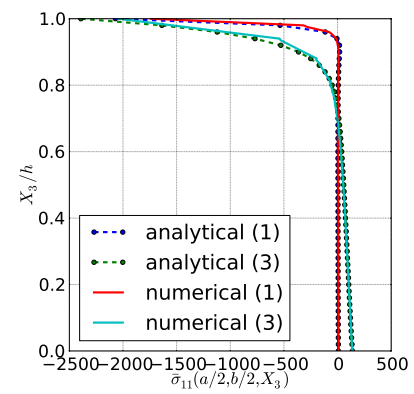

(b)
Figure 7: Normalized normal stress distribution $\bar{\sigma}_{11}$ over the normalized depth at the center of the plate for plates 1 and 2 (left) and 1 and 3 (right). The numerical solution is shown in continuous lines whereas the analytical solution is depicted using dashed lines.

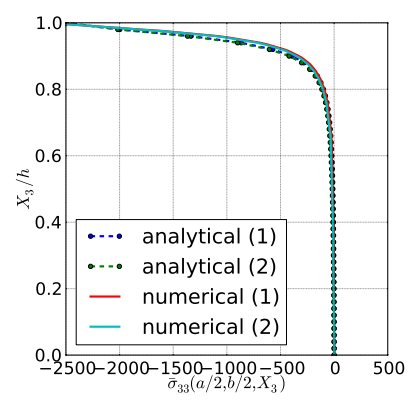

(a)

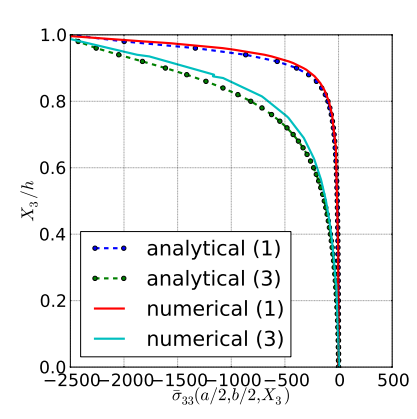

(b)
Figure 8: Normalized normal stress distribution $\bar{\sigma}_{33}$ over the normalized depth at the center of the plate for plates 1 and 2 (left) and 1 and 3 (right). The numerical solution is shown in continuous lines whereas the analytical solution is depicted using dashed lines.

The numerical method herein can be generalized for any other geometry and loading condition, moreover the proposed a posteriori error estimation based on the equivalent stress measure appears to be adequate for many engineering problems.

\section{Conclusions}

An analytical solution and numerical approximation by using finite element method has been presented that allows for the computation of stresses and displacements of a functionally graded, three-dimensional continuum under point loading. The point load is simulated by the 
FOURIER series in the analytic solution and by an equivalent GAUssian distribution in the numerical approximation in order to circumvent numerical errors due to GIBBS's phenomena. Three different plate geometries were implemented for validation purposes. The difficulties for an optimum choice of the element size has been discussed. By using a posteriori error estimation based on the equivalent stress measure quantitatively accurate results have been obtained - even in the neighborhood of the point loading. The computational work has been realized by using open-source program packages. The code for the applications herein has been published in Abali (2011-) under the GNU public license as in Gnu Public (2007). Since good agreement between the analytical and numerical solution was achieved, the numerical approach can now be used to analyze more complex structural shapes and components comprising isotropic, functionally graded properties.

Abali, B.E., 2011-. Technical University of Berlin, Institute of Mechanics, Chair of Continuums Mechanics and Material Theory, Computational Reality. http://www.lkm.tuberlin.de/ComputationalReality/.

Abali, B.E., Völlmecke, C., Woodward, B., Kashtalyan, M., Guz, I., Müller, W.H., 2012. Numerical modeling of functionally graded materials using a variational formulation. Continuum Mechanics and Thermodynamics 24, 377-390.

Birman, V., Byrd, L.W., 2007. Modeling and analysis of functionally graded materials and structures. Applied Mechanics Reviews 60, 195-216.

Doddamani, M.R., Kulkarno, S.M., Kishore, 2011. Behaviour of sandwich beams with functionally graded rubber core in three point bending. Polymer Composites , 1541-1551.

Gibbs, J.W., 1898. Fourier's series. Nature 59, 200.

Gibbs, J.W., 1899. Fourier's series. Nature 59, 606.

Gnu Public, 2007. Gnu general public license. http://www.gnu.org/copyleft/gpl.html.

Guo, L.C., Wu, L.Z., Ma, L., 2004. The interface crack problem under a concentrated load for a functionally graded coating-substrate composite system. Composite Structures 63, 397-406.

Henderson, A., 2007. ParaView Guide, A Parallel Visualization Application, Kitware Inc. http://paraview.org/.

Hilbert, D., 1902. (transl. by E. J. Townsend), The Foundations of Geometry. The Open Court Publishing Co. .

Hoffman, J., Jansson, J., Johnson, C., Knepley, M., Kirby,
R., Logg, A., Scott, L.R., Wells, G.N., 2005. Fenics. http://www.fenicsproject.org/.

Hunter, J.D., 2007. Matplotlib: A 2d graphics environment. Computing In Science \& Engineering 9, 90-95.

Kashtalyan, M., 2004. Three-dimensional elasticity solution for bending of functionally graded rectangular plates. European Journal of Mechanics-A/Solids 23, 853-864.

Logg, A., Mardal, K.A., Wells, G.N., 2011. Automated Solution of Differential Equations by the Finite Element Method, The FEniCS Book. volume 84 of Lecture Notes in Computational Science and Engineering. Springer.

Logg, A., Wells, G.N., 2010. Dolfin: Automated finite element computing. ACM Transactions on Mathematical Software 37.

Müller, W.H., 2011. Streifzüge durch die Kontinuumstheorie. Springer.

Pagano, N., 1969. Exact solutions for composite laminates in cylindrical bending. Journal of Composite Materials 3, 398-411.

Singh, J., Shukla, K.K., 2012. Nonlinear flexural analysis of functionally graded plates under different loading using RBF based meshless method. Engineering Analysis with Boundary Elements 36, 1819-1827.

Spencer, A.J.M., 2000. Concentrated force solutions for an inhomogeneous thick elastic plate. Zeitschrift für angewandte Mathematik und Physik ZAMP 51, 573-590.

Sun, D., Luo, S.N., 2011a. Wave propagation and transient response of a FGM plate under a point impact load based on higher-order shear deformation theory. Composites Structures 93, 1474-1484.

Sun, D., Luo, S.N., 2011b. Wave propagation and transient response of functionally graded material circular plates under a point impact load. Composites: part B 42, 657-665.

Sun, D., Luo, S.N., 2012. Wave propagation and transient response of a functionally graded material plate under a point impact load in thermal environments. Applied Mathematical Modelling 44, 369-462.

Wang, C.D., Tzeng, C.S., Pan, E., Liao, J.J., 2003. Displacements and stresses due to a vertical point load in an inhomogeneous transversely isotropic half-space. International Journal of Rock Mechanics and Mining Science 40, 667-685.

Woodward, B., Kashtalyan, M., 2011. 3D elasticity analysis of sandwich panels with graded core under distributed and concentrated loadings. International Journal of Mechanical Sciences 53, 872885.

Woodward, B., Kashtalyan, M., 2012. Performance of functionally graded plates under localised transverse loadings. Composite Structures 94, 2254-2262. 


\section{Appendix A. Appendix}

The functions $U_{i, k}$ for the displacements are:

$$
\begin{gathered}
U_{1, j m n}\left(\bar{X}_{3}\right)=-\frac{q_{m n} h}{2 G_{1}} \frac{\pi m h}{a} \exp \left(-\gamma\left(\bar{X}_{3}-1\right)\right) \times \\
\times\left(-\nu \alpha_{m n}^{2} h^{2} f_{j m n}\left(\bar{X}_{3}\right)+(\nu-1) \frac{\mathrm{d}^{2}}{\mathrm{~d} \bar{X}_{3}^{2}} f_{j m n}\left(\bar{X}_{3}\right)\right), \\
j=1,2,3,4 ; \\
U_{1, j m n}\left(\bar{X}_{3}\right)=-\frac{q_{m n} h}{G_{1}} \frac{\pi n h}{b} f_{j m n}\left(\bar{X}_{3}\right), j=5,6 ; \\
U_{2, j m n}\left(\bar{X}_{3}\right)=-\frac{q_{m n} h}{2 G_{1}} \frac{\pi n h}{b} \exp \left(-\gamma\left(\bar{X}_{3}-1\right)\right) \times \\
\times\left(-\nu \alpha_{m n}^{2} h^{2} f_{j m n}\left(\bar{X}_{3}\right)+(\nu-1) \frac{\mathrm{d}^{2}}{\mathrm{~d} \bar{X}_{3}^{2}} f_{j m n}\left(\bar{X}_{3}\right)\right), \\
U_{2, j m n}\left(\bar{X}_{3}\right)=-\frac{q_{m n} h}{G_{1}} \frac{\pi m h}{a} f_{j m n}\left(\bar{X}_{3}\right), j=5,6 ; \\
U_{3, j m n}\left(\bar{X}_{3}\right)=-\frac{q_{m n} h}{2 G_{1}} \frac{\pi m h}{a} \exp \left(-\gamma\left(\bar{X}_{3}-1\right)\right) \times \\
\times\left((\nu-1)\left(-\gamma \frac{\mathrm{d}^{2}}{\mathrm{~d} \bar{X}_{3}^{2}} f_{j m n}\left(\bar{X}_{3}\right)+\frac{\mathrm{d}^{2}}{\mathrm{~d} \bar{X}_{3}^{2}} f_{j m n}\left(\bar{X}_{3}\right)\right)-\right. \\
\left.-\alpha_{m n}^{2} h^{2}\left((\nu-2) d d \bar{X}_{3} f_{j m n}\left(\bar{X}_{3}\right)-\nu \gamma f_{j m n}\left(\bar{X}_{3}\right)\right)\right), \\
j=1,2,3,4 ; \\
U_{3, j m n}\left(\bar{X}_{3}\right)=0, j=5,6 ;
\end{gathered}
$$

For stresses, functions $P_{i j, k}$ are found to be as follows

$$
\begin{aligned}
& P_{11, j m n}\left(\bar{X}_{3}\right)=q_{m n}\left(\nu \alpha_{m n}^{2} h^{2}\left(\frac{\pi n h}{b}\right)^{2} f_{j m n}\left(\bar{X}_{3}\right)-\right. \\
&-\nu\left(\frac{\pi n h}{b}\right)^{2} \frac{\mathrm{d}^{2}}{\mathrm{~d} \bar{X}_{3}^{2}} f_{j m n}\left(\bar{X}_{3}\right)- \\
&\left.-\left(\frac{\pi m h}{a}\right)^{2} \frac{\mathrm{d}^{2}}{\mathrm{~d} \bar{X}_{3}^{2}} f_{j m n}\left(\bar{X}_{3}\right)\right), j=1,2,3,4 ; \\
& P_{11, j m n}\left(\bar{X}_{3}\right)=2 q_{m n} \frac{\pi n h}{b} \frac{\pi m h}{a} \exp \left(\gamma\left(\bar{X}_{3}-1\right)\right) \times \\
& \times f_{j m n}\left(\bar{X}_{3}\right), j=5,6 ;
\end{aligned}
$$

$$
\begin{gathered}
P_{22, j m n}\left(\bar{X}_{3}\right)=q_{m n}\left(\nu \alpha_{m n}^{2} h^{2}\left(\frac{\pi m h}{a}\right)^{2} f_{j m n}\left(\bar{X}_{3}\right)-\right. \\
-\nu\left(\frac{\pi m h}{a}\right)^{2} \frac{\mathrm{d}^{2}}{\mathrm{~d} \bar{X}_{3}^{2}} f_{j m n}\left(\bar{X}_{3}\right)- \\
\left.-\left(\frac{\pi n h}{b}\right)^{2} \frac{\mathrm{d}^{2}}{\mathrm{~d} \bar{X}_{3}^{2}} f_{j m n}\left(\bar{X}_{3}\right)\right), j=1,2,3,4 ;
\end{gathered}
$$$$
P_{22, j m n}\left(\bar{X}_{3}\right)=-2 q_{m n} \frac{\pi n h}{b} \frac{\pi m h}{a} \exp \left(\gamma\left(\bar{X}_{3}-1\right)\right) \times
$$$$
\times f_{j m n}\left(\bar{X}_{3}\right), j=5,6 \text {; }
$$$$
P_{33, j m n}\left(\bar{X}_{3}\right)=q_{m n} \alpha_{m n}^{4} h^{4} f_{j m n}\left(\bar{X}_{3}\right), j=1,2,3,4 ;
$$$$
P_{33, j m n}\left(\bar{X}_{3}\right)=0, j=5,6 ;
$$$$
P_{13, j m n}\left(\bar{X}_{3}\right)=q_{m n} \alpha_{m n}^{2} h^{2} \frac{\pi m h}{a} \frac{\mathrm{d}}{\mathrm{d} \bar{X}_{3}} f_{j m n}\left(\bar{X}_{3}\right),
$$

$$
j=1,2,3,4 \text {; }
$$

$$
\begin{gathered}
P_{13, j m n}\left(\bar{X}_{3}\right)=-q_{m n} \frac{\pi n h}{b} \exp \left(\gamma\left(\bar{X}_{3}-1\right)\right) \frac{\mathrm{d}}{\mathrm{d} \bar{X}_{3}} f_{j}\left(\bar{X}_{3}\right), \\
j=5,6 ;
\end{gathered}
$$

$$
P_{23, j m n}\left(\bar{X}_{3}\right)=q_{m n} \alpha_{m n}^{2} h^{2} \frac{\pi n h}{b} \frac{\mathrm{d}}{\mathrm{d} \bar{X}_{3}} f_{j m n}\left(\bar{X}_{3}\right),
$$$$
j=1,2,3,4 ;
$$$$
P_{23, j m n}\left(\bar{X}_{3}\right)=q_{m n} \frac{\pi m h}{a} \exp \left(\gamma\left(\bar{X}_{3}-1\right)\right) \frac{\mathrm{d}}{\mathrm{d} \bar{X}_{3}} f_{j m n}\left(\bar{X}_{3}\right) \text {, }
$$

$$
j=5,6 \text {; }
$$

$$
\begin{gathered}
P_{12, j m n}\left(\bar{X}_{3}\right)=q_{m n} \frac{\pi m h}{a} \frac{\pi n h}{b}\left(\nu \alpha_{m n}^{2} h^{2} f_{j m n}\left(\bar{X}_{3}\right)+\right. \\
\left.+(1-\nu) \frac{\mathrm{d}^{2}}{\mathrm{~d} \bar{X}_{3}^{2}} f_{j m n}\left(\bar{X}_{3}\right)\right), j=1,2,3,4 ; \\
P_{12, j m n}\left(\bar{X}_{3}\right)=q_{m n}\left(\left(\frac{\pi m h}{a}\right)^{2}-\left(\frac{\pi n h}{b}\right)^{2}\right) \times \\
\times \exp \left(\gamma\left(\bar{X}_{3}-1\right)\right) f_{j m n}\left(\bar{X}_{3}\right), j=5,6
\end{gathered}
$$


In the expressions above, $\bar{X}_{3}=X_{3} / h$, and functions $f_{j m n}\left(\bar{X}_{3}\right)$, $j=1, \ldots, 6$ are:

$$
\begin{gathered}
f_{1 m n}\left(\bar{X}_{3}\right)=\exp \left(\frac{\gamma \bar{X}_{3}}{2}\right) \cosh \left(\lambda_{m n} \bar{X}_{3}\right) \cos \left(\mu \bar{X}_{3}\right), \\
f_{2 m n}\left(\bar{X}_{3}\right)=\exp \left(\frac{\gamma \bar{X}_{3}}{2}\right) \sinh \left(\lambda_{m n} \bar{X}_{3}\right) \cos \left(\mu \bar{X}_{3}\right), \\
f_{3 m n}\left(\bar{X}_{3}\right)=\exp \left(\frac{\gamma \bar{X}_{3}}{2}\right) \cosh \left(\lambda_{m n} \bar{X}_{3}\right) \sin \left(\mu \bar{X}_{3}\right), \\
f_{4 m n}\left(\bar{X}_{3}\right)=\exp \left(\frac{\gamma \bar{X}_{3}}{2}\right) \sinh \left(\lambda_{m n} \bar{X}_{3}\right) \sin \left(\mu \bar{X}_{3}\right), \\
f_{5 m n}\left(\bar{X}_{3}\right)=\exp \left(-\frac{\gamma \bar{X}_{3}}{2}\right) \cosh \left(\beta_{m n} \bar{X}_{3}\right), \\
f_{6 m n}\left(\bar{X}_{3}\right)=\exp \left(-\frac{\gamma \bar{X}_{3}}{2}\right) \sinh \left(\beta_{m n} \bar{X}_{3}\right) .
\end{gathered}
$$

